\title{
Case Report \\ Importance of Video Technologies in Seizure Identification - Evidence through a Case Report
}

\author{
João Rocha and João Pereira \\ Neurology Department - Hospital de Braga, Sete Fontes - S. Victor, Portugal
}

Received 3 March 2013; Accepted 8 April 2013; Published 20 May 2013

Academic Editor: Péter Halász

Copyright (C 2013 João Rocha and João Pereira. Distributed under Creative Commons CC-BY 3.0

\begin{abstract}
Epilepsy is characterized by unprovoked repetitive occurrence of seizures, which are the clinical manifestation of an abnormal cerebral discharge. Seizure semiology is widely variable, in some cases being difficult to identify and diagnose. Especially in non-convulsive seizures, a high grade of suspicion is necessary and description of episodes by patients and witness may be bizarre and misleading. Video recording of seizures could be widely available through portable cameras and may help physicians recognize seizures. We present a case of a patient with an eight years history of non-convulsive seizures that was only diagnosed with epilepsy after self recording a seizure on his phone camera. Through this case we would like to alert the medical community for the usefulness of these widespread portable video technologies in the diagnosis of seizure disorders.
\end{abstract}

Keywords: Epilepsy; Non-convulsive seizures; Video.

\section{Introduction}

Since the advent of electroencephalography, one of the major developments in the area of neurophysiologic study was the introduction of video co-registering. This tool is especially important in the diagnosis and differentiation of epileptic seizures from non-epileptic seizuresas reported by McGonigal et al (2004) and Raymond et al (1999). The diagnosis of epilepsy is clinical, by recognition of recurring unprovoked seizures, dependent upon a very detailed history as mentioned by Jan (2008). Seizure description by patients' or witnesses' recollection is a key point in epilepsy study and recording of the chronological sequence of recurrent, transient, self-limited, alteration in neurological state, must be meticulously sought. The quality of these inquiries allows understanding the patient's complaints and assist on the diagnosis of epilepsy, helps classifying the epileptic syndrome and localizing a possible epileptic focus. This last aspect is most important in non-convulsive seizures, as in temporal lobe epilepsy as previously described by Jan and Girvin (2008). A hand held video camera may provide an accurate description of a seizure, which is often clearer and sometimes different from that which is obtained from an eye witness account, as even experienced medical and nursing staff may have difficulty in describing seizures. With the advent and evolution of portable video cameras, such as those integrated on mobile phones, there has been an intuitively recognized usefulness of this technology in seizures study. One should note that some complex

Cite this Article as: João Rocha and João Pereira (2013), "Importance of Video Technologies in Seizure Identification - Evidence through a Case Report," International Journal of Case Reports in Medicine, Vol. 2013 (2013), Article ID 656341, DOI: 10.5171/2013. 656341 
partial seizures may appear bizarre, and interpretation based on patient or witness recollection, even by experienced observers may not be straightforward, leading to delays in epilepsy diagnosis. Hence, video registry could be an important addition and must be assessed in conjunction with the history, image studies and EEG (Samuels and Duncan (1994)).

We report a patient with a history of 8 years of transient changes in his neurological state, whose diagnosis of epilepsy had been missed until he was able to self record a seizure with his phone camera.

\section{Case Report}

A 28-year-old, ex-military male was referred to our Department due to transient episodes of loss of consciousness. He had no personal or family history of epilepsy, no febrile seizures, neurologic infections or significant head traumas.

He complaint of transient episodes of loss of consciousness since he was 20 years-old. He referred a prodromal sensation of ascending gastric discomfort followed by right upper limb involuntary and repetitive movements, and progression in less than one minute to suppression of consciousness. His wife witnessed some episodes. She reported that the patient has his eyes opened during the episode and was unresponsive to external stimuli. He had no speech for about one minute, and only when asked reports simultaneous chewing movements. He has no memory for this period. Subsequently, he regains consciousness, is well aware of the surrounding and recalls this phase, but presents a ten minute period of apparent difficulty understanding and producing speech with complete recovery, which was interpreted as a transient aphasia. No convulsive activity was ever observed nor loss of body tonus or sphincter control. These episodes would occur in clusters of two to six episodes during three days.
Initially he had one cluster per year but lately frequency had increased with episodes every month. He inclusively had had a traffic accident during a seizure.

He had already consulted several physicians and performed cerebral CT scan and electroencephalograms (EEG) which were normal. No episodes had ever occurred during his medical visits, and due to his military background, he was diagnosed with post-traumatic stress.

After eight years of symptoms he was able to self record a seizure on his phone camera. After experiencing the epigastric aura, the patient activated the recording function and placed the camera on the desk pointing at him. In the recording we can observe the clinical details that had been poorly described. He presented exuberantrightarm automatisms with circular movements and tapping on the desk, and almost concomitantly sudden loss of visual contact, occasional blinking and oral automatisms with chewing movements. After about a minute the automatisms stop, the patient seems confused and does not speak and stopped the recording. After presenting this video to another doctor, he came to our Department. Semiologically these seemed mesial temporal lobe seizures and we decided to perform cerebral magnetic resonance (MR) and repeat video-EEG. EEG was again normal, but he was asymptomatic during the recording. MR revealed loss of cortical-subcortical differentiation in the left hippocampal formation, associated to signal T2 hyperintensity in the hippocampal body and tail suggesting mesial sclerosis. Right hippocampus was normal and no other pathological changes were found (Figure 1).

He was started on oxcarbazepine with slow increase of dose until $1200 \mathrm{mg} /$ daily with excellent response, being seizure free for a year. 


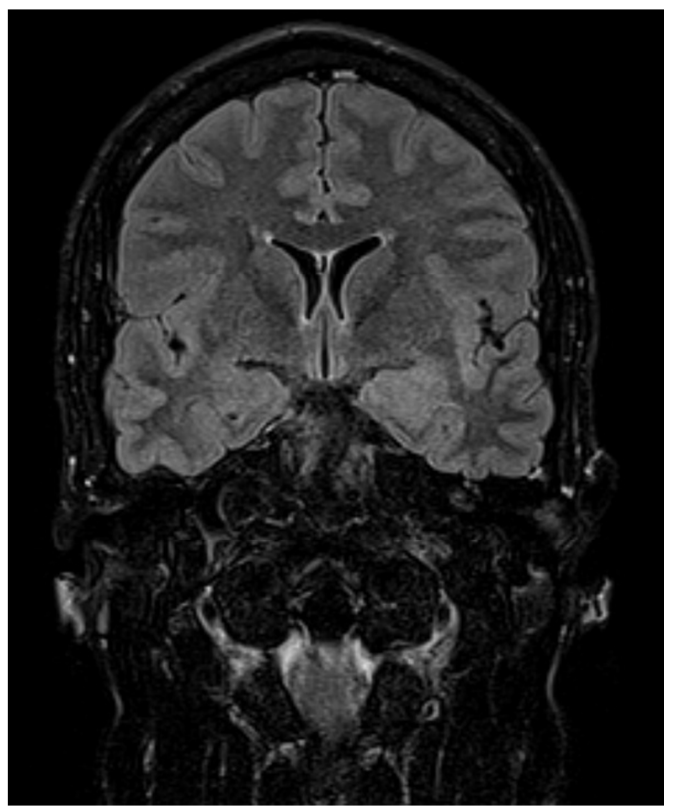

\section{Figure 1: MRI - T2 FLAIR Coronal Section with Loss of Cortical-Subcortical Differentiation and Hyperintensity in Left Hippocampal Formation Suggesting Mesial Temporal Sclerosis}

\section{Discussion}

With this case we would like to highlight the importance that video recording had in the diagnosis of our patient. For eight years he had been having seizures that were a source of anxiety and were limiting his quality of life and safety.

Clinically, these were mesial temporal focal seizures, with an epigastric aura followed by suppression of consciousness, limb and oral automatisms and eye blinking. The post-ictalaphasia indicated a probable dominant-lobe temporal focus. Although, semiologically typical, they are difficult to describe by patients, due to their lack of awareness during the episode, and for a lay witness, some details such as the chewing or blinking could seem irrelevant. Also, the fact that our patient's seizures were brief, and at first widely spaced in time, made difficult any observation by the physicians that were less trained in the area of epilepsy. As reported by Bronen et al (1996), in temporal sclerosis CT scan may be irrelevant and inter-ictal EEG normal according to Cersósino et al (2011). However, epilepsy diagnosis is clinical.
Our patient is a paradigm of a focal temporal lobe epilepsy, whose diagnosis had been missed due to the absence of convulsive activity and presence of subtle clinical aspects that were only identified after video recording.

With recent diffusion of portable video recording technologies such as in mobile phones, we suggest that, in cases where there is description of transient alterations in neurologic status that cannot be accurately characterized, we should encourage patients and caregivers to record these events, in order to increase our capacity to identify and detail seizures.

\section{References}

Bronen, R. A., Fulbright, R. K., Spencer D. D., et al. (1996). "Refractory Epilepsy: Comparison of MR Imaging, CT, and Histopathologic Findings in 117 Patients," Radiology, 201 (1): 97-105.

Cersósimo, R., Flesler, S., Bartuluchi, M., et al. (2011). "Mesial Temporal Lobe Epilepsy with Hippocampal Sclerosis: Study of 42 Children," Seizure, 20: 131-137. 
Jan, M. M. (2008). 'Clinical Review of Pediatric Epilepsy,' Neuroscience, 10: 255264.

Jan, M. M. S. \& Girvin, J. P. (2008). "Seizure Semiology: Value in Identifying Seizure Origin," Canadian Journal of Neurological Sciences, 35: 22-30.

McGonigal, A., Russell, A. J. C., Mallik, A. K., et al. (2004). "Use of Short Term Video EEG in the Diagnosis of Attack Disorders," Journal of Neurology, Neurosurgery \& Psychiatry, 75: 771-772.

Raymond, A. A., Gilmore, W. V., Scott, C. A., Fish, D. R. \& Smith, S. J. (1999). "Video-EEG Telemetry: Apparent Manifestation of Both Epileptic and Non-Epileptic Attacks Causing Potential Diagnostic Pitfalls," Epileptic Disorders, 1(2): 101-106.

Samuels, M. \& Duncan, J. S. (1994). “Use of Hand Held Video Camcorder in the Evaluation of Seizures," Journal of Neurology, Neurosurgery \& Psychiatry, 57: 1417-1418. 